\title{
Simultaneous antegrade urography of the upper urinary tract and retrograde cystography combined with computed tomography imaging in the management of ureteral complications after renal transplantation
}

\author{
Lei Zhang ${ }^{1,2 \#}$, Luhao Liu ${ }^{1 \#}$, Xingqiang Lai ${ }^{1 *}$, Jiali Fang ${ }^{1}$, Yuhe Guo ${ }^{1}$, Guanghui Li $^{1}$, Lu Xu ${ }^{1}$, Yunyi Xiong ${ }^{1}$, \\ Wei Yin ${ }^{1}$, Junjie $\mathrm{Ma}^{1}$, Zheng Chen ${ }^{1,2}$ \\ ${ }^{1}$ Department of Organ Transplantation, The Second Affiliated Hospital of Guangzhou Medical University, Guangzhou, China; ${ }^{2}$ Department of \\ Organ Transplantation, The Second Clinical Medicine School of Guangzhou Medical University, Guangzhou, China \\ Contributions: (I) Conception and design: L Zhang, Z Chen; (II) Administrative support: Z Chen; (III) Provision of study materials or patients: L Liu; \\ (IV) Collection and assembly of data: L Zhang, Z Chen, L Liu, X Lai, J Fang, Y Guo, Y Xiong, W Yin, J Ma; (V) Data analysis and interpretation: L \\ Liu, X Lai, J Fang, Y Guo, G Li, L Xu, W Yin, J Ma; (VI) Manuscript writing: All authors; (VII) Final approval of manuscript: All authors. \\ \#These authors contributed equally to this work. \\ Correspondence to: Zheng Chen, MD, PhD. Department of Organ Transplantation, The Second Affiliated Hospital of Guangzhou, Medical University, \\ Guangzhou, China. Email: docchenzheng@163.com.
}

Background: To investigate the significance of simultaneous urography of the upper and lower urinary tract of transplanted kidneys combined with computed tomography urography (CTU), computed tomography arteriography (CTA), and computed tomography venography imaging in the planning of open surgery performed to treat any ureteral complications of a transplanted kidney.

Methods: In all, 24 patients with ureteral complications after renal transplantation were admitted, 12 of whom had renal graft ostomy during open surgery. Simultaneous antegrade urography of the upper urinary tract and retrograde cystography of the transplanted kidneys were performed on the patients. With the use of computed tomography imaging results, surgical planning was carried out.

Results: All surgeries were successfully completed according to preoperative planning. Three patients underwent end-to-end anastomosis of the ureter and bladder muscle flap, 8 patients underwent ureterocystostomy, and 1 patient underwent an end-to-end ureteral anastomosis. After the follow-up up to now, all the patients had stable renal function, and no complications such as ureteral stenosis or urine leakage have thus far reoccurred in the transplanted kidneys.

Conclusions: When open surgery is required to treat any ureteral complications following renal transplantation, preoperative multiangle imaging can be used to better understand the condition of the transplanted urinary tract and thus aid considerably in surgical planning.

Keywords: Renal transplantation; ureteral complications; antegrade urography of the upper urinary tract; retrograde cystography; bladder muscle flap

Submitted Jun 09, 2021. Accepted for publication Sep 15, 2021.

doi: 10.21037/tau-21-614

View this article at: https://dx.doi.org/10.21037/tau-21-614 


\section{Introduction}

Ureteral complications after renal transplantation are relatively common, with national and international incidence rates reported to be $1 \%$ to $8 \%(1,2)$. The main treatments for these complications are intracavitary therapy and open surgery, with the main goals being the reconstruction of the transplanted kidney's urinary tract, which thus frees the patient from undergoing a renal graft ostomy and the insertion of a long-term indwelling double $\mathrm{J}$ stent. The open surgery failure rate has been reported to be $7 \%$ to $8 \%(3,4)$, with multiple reasons for these failures evidenced, for example, inadequate preoperative preparation (5). This article aims to summarize the author's clinical experiences related to selecting the most appropriate preoperative surgical plan for a successful surgery. We present the following article in accordance with the STROBE reporting checklist (available at https://dx.doi. org/10.21037/tau-21-614).

\section{Methods}

\section{Study population}

From January 2016, 2020, to July 2020, 1,338 kidney transplants by citizen organ donation, 141 simultaneous pancreas-kidney transplants (SPKTs), and 1 simultaneous liver-kidney transplant (SLKT) were included as study subjects and retrospectively reviewed in our center. All transplants involved in this study were reviewed and approved by the hospital's ethics committee.

During this period, 24 patients with ureteral complications after renal transplantation were admitted. Among them, 16 patients had ureteral obstruction and 8 had urinary leakage. There were 18 kidney transplant recipients, 5 SPKTs, and 1 SLKT. Additionally, 12 patients underwent renal graft ostomy with open surgery, with 11 renal transplant recipients and 1 SLKT recipient.

\section{Ethical statement}

The study was conducted in accordance with the Declaration of Helsinki (as revised in 2013). The study was approved by The Institutional Review Board of the Second Affiliated Hospital of Guangzhou Medical University (number 2020-hs-43) and informed consent was taken from all individual participants.

\section{Imaging}

All 12 patients had preoperative renal graft ostomy and underwent simultaneous urography of the upper and lower urinary tract once their conditions had stabilized. In the hospital wards, a 16-gauge catheter was inserted into the patients following intraurethral anesthesia with tetracaine administration. In the radiology department under aseptic conditions, the contrast agent, iodixanol (Visipaque $270 \mathrm{mg} / \mathrm{mL}$ ), diluted with 1:1 saline, was slowly injected into the renal graft fistula: patients with a ureteral obstruction were injected with $10 \mathrm{~mL}$, and those with urinary leakage were injected with $20 \mathrm{~mL}$. After a satisfactory imaging from X-ray fluoroscopy, the fistula was clamped and the contrast agent was simultaneously retrograde injected into bladder through the catheter. The injection was stopped when a patient complained of bladder distension, and the dose of contrast agent injected into the bladder was recorded. The urinary tracts of the transplanted kidneys were photographed from different angles under X-ray fluoroscopy, as shown in Figure 1.

\section{Assessment of surgical feasibility and formulation of surgical plan}

To determine whether the patient's own ureter on the same side of the transplanted kidney was unobstructed, a retrograde ureteral catheter was routinely placed under cystoscopy before surgery.

The surgical plan for each patient was devised based on preoperative radiography, with 3 widely used surgical procedures being adopted for reconstruction of the transplanted kidney's urinary tract: ureterovesical muscle flap anastomosis, ureterocystostomy, and renal graft ureter to the patient's own ureteral anastomosis $(6,7)$. If preoperative radiography showed a bladder volume greater than $200 \mathrm{~mL}$, greater bladder filling could be obtained after administration of anesthesia. Ureteral bladder reimplantation could be performed if the retained renal graft ureter was long, but if the retained ureter was short, ureterovesical muscle flap anastomosis could be performed with greater confidence. Ureteral bladder reimplantation could still be performed if the bladder volume was less than $100 \mathrm{~mL}$ and the retained ureter was long, but if the retained ureter was short and the bladder muscle flap anastomosis was difficult to achieve, end-to-end ureter anastomosis to a patient's own ureter could be achieved. 

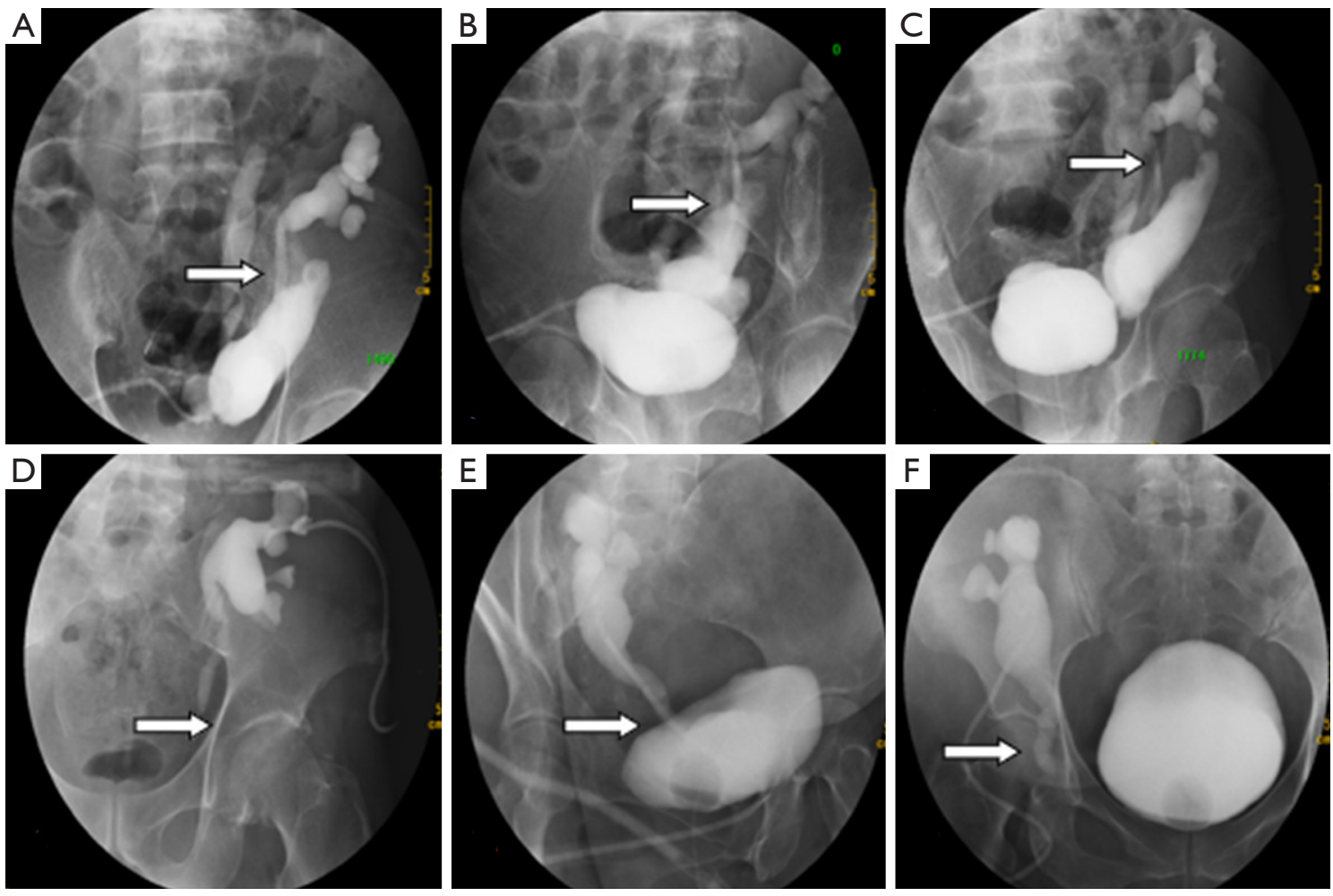

Figure 1 Preoperative simultaneous antegrade urography of upper urinary tract and retrograde cystography. (A,D) Show radiography of the upper urinary tract. (B,C,E,F) Show simultaneous urography of the upper and lower urinary tract. The white arrows indicate the ureteral stump.

\section{Ureterovesical muscle flap anastomosis of the transplanted Kidney (Boari flap)}

This procedure was used when the bladder volume was not ideal and the retained ureter was short. The ureteral surface projection of the transplanted kidney was marked based on computed tomography urography (CTU) imaging. After pushing aside and folding the peritoneum, the bladder was fully freed and the ureter was cut. The original anastomosis stoma was then sutured, and the full layer of bladder tissue $5 \mathrm{~cm} \times 3 \mathrm{~cm}$ was collected for the Boari flap. The proximal ureter of the transplanted kidney was anastomosed with the bladder muscle flap end to end using a built-in F6 double J-tube without thread, and then relaxation suture was applied to the surrounding tissue, as shown in Figure 2.

\section{Ureteroneocystostomy of the transplanted kidney}

This procedure was carried out when the retained ureter of the transplanted kidney was long. Exploration of the ureter and management of the bladder were undertaken using these same methods. After removal of the necrotic or obstructed portion, the proximal ureter was anastomosed to the bladder with a built-in F6 double J tube without thread, and then relaxation suture was applied to the surrounding tissue

\section{End-to-end anastomosis between the transplanted renal ureter and its own distal ureter}

This procedure was used when the retained ureter from the transplanted kidney was short and the bladder was poorly filled. Exploration of the ureter and management of the bladder were performed as previously described. The proximal end of the patient's ureter was dislocated and ligated, and the distal end was left with a sufficient length to be anastomosed end to end, with the repaired of the ureter and a built-in F6 double J tube without thread.

\section{Postoperative management}

Urinary tubes and iliac fossa drainage tubes were routinely 

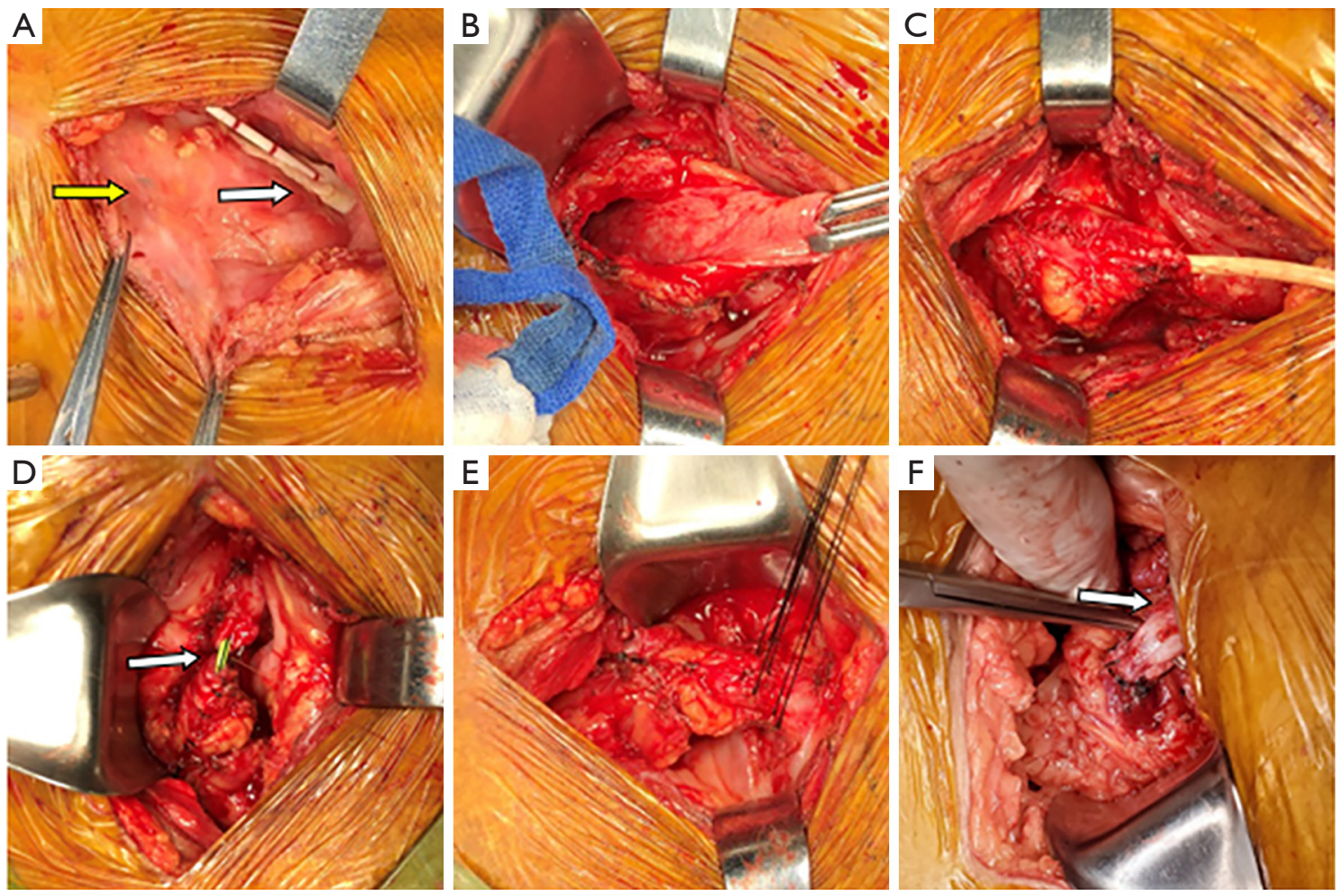

Figure 2 Ureterovesical muscle flap anastomosis of the transplanted kidney. The white arrows point to the ureteral stump of the transplanted kidney in (A,D,F). The yellow arrow points to the area of the urinary cyst mentioned in (A).

indwelt during the operation. Postoperatively, fluid was observed draining from the transplanted kidney's fistula, the iliac fossa drainage tube, and the urinary duct, and any urinary leakage or obstruction was observed with color Doppler ultrasound of the transplanted kidney. In the absence of any special conditions, the fistula and iliac fossa drainage tubes were removed about a week, the urinary tube after 2 weeks, and the double J tube 1 month after surgery.

\section{Statistical analysis}

Data were analyzed using SPSS 16.0 software (IBM Corp., Armonk, NY, USA) Continuous data are expressed as mean \pm standard deviation, and a paired $t$-test was used for comparison. All $\mathrm{P}$ values were 2 tailed, and a $\mathrm{P}$ value $<0.05$ was considered statistically significant.

\section{Results}

\section{Baseline data}

Ureteral complications after renal transplantation were generally found 1 to 9 months after surgery (a mean of 2.5 months). Among them, there were 7 males and 5 females, aged 28 to 64 years, with an average age of $42.0 \pm 9.9$ years. The early postoperative period saw 2 cases of acute rejection and 4 cases of delayed graft function (DGF). Among the 12 patients, there were 7 cases of ureteral obstruction and 5 of urinary leakage. Each patient's bladder was injected with 60 to $340 \mathrm{~mL}$ of contrast medium, with an average of $210.8 \pm 92.4 \mathrm{~mL}$. The procedures were completed successfully according to preoperative planning, with 3 patients undergoing end-to-end ureterovesical muscle flap anastomosis, 8 patients having a ureterocystostomy, and 1 patient having end-to-end ureter anastomosis to their own ureter. Details are shown in Table 1.

\section{Surgical efficacy}

All patients underwent successful postoperative removal of the renal graft fistula, iliac fossa drainage tube, urinary tube, and double J tube. Stable phase serum creatinine levels after transplantation and before the operation were $159.8 \pm 57.6$ 
Table 1 Baseline data

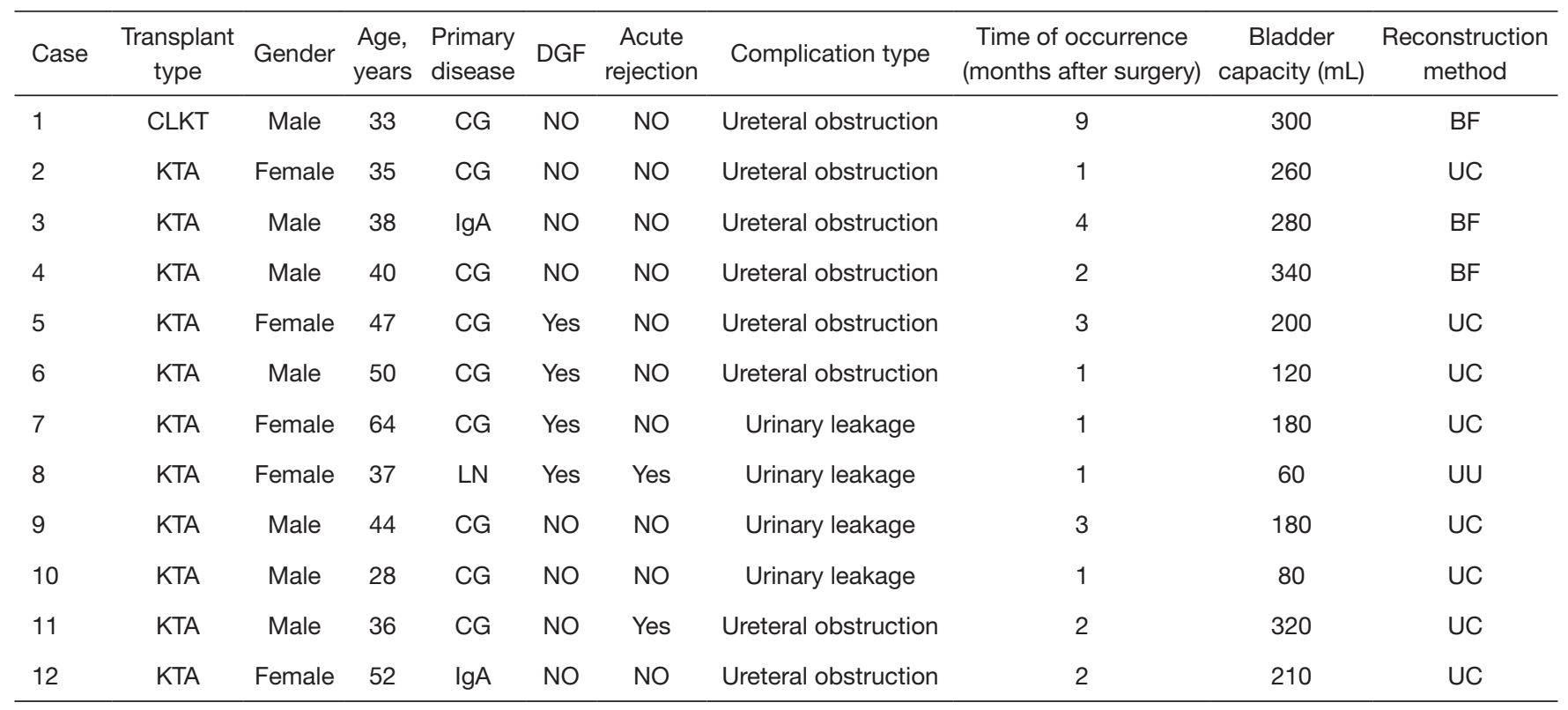

BF, Boari flap; CLKT, combined liver and kidney transplantation; CG, chronic glomerulonephritis; KTA, kidney transplant alone; LN, lupus nephritis; NO, not applicable; UC, ureterocystostomy; UU, ureteroureterostomy.

and $387.9 \pm 272.7$, respectively $(\mathrm{P}=0.012)$, with the serum creatinine before patient discharge being $151.2 \pm 62.4 \mu \mathrm{mol} / \mathrm{L}$. There were no significant differences between at discharge and stable phases after renal transplantation in renal allograft function $(\mathrm{P}=0.319)$. To reduce further damage of graft function caused by contrast agents, we used iodixanol injection (Visipaque) as the contrast agent in all cases. The patients serum creatinine levels before and after injection of the contrast medium were respectively $228.4 \pm 165.1 \mu \mathrm{mol} / \mathrm{L}$ and $230.2 .9 \pm 173.2 \mu \mathrm{mol} / \mathrm{L}(\mathrm{P}=0.746)$.

The patients were followed up for an average of 21 months (2-56 months), and no follow-up appointments were missed. Renal function was stable in all patients except 1, who, due to BK virus-associated transplant nephropathy (BKVAN), had a higher serum creatinine level than that prior to the procedure. Postoperative color Doppler ultrasound or urinary tract CTU of the transplanted kidneys were performed after 1 month, 6 months, 1 year after surgery and annually thereafter, and no reobstruction or urinary leakage was detected. Mild vesicoureteral reflux was present in 1 patient, but no special management was required.

\section{Complications of reoperation}

The patients in this groups recovered well after surgery. No anastomotic stenosis or urinary leakage were evident in the color Doppler ultrasound and CTU re-examination of the transplanted kidneys nor were there any visible renal graft or vascular injuries.

\section{Follow-up}

The patients were followed up for an average of 21 months, and no follow-up appointment went unattended. Renal function was stable in all patients except the 1 patient with BKVAN, who had a higher serum creatinine level than that recorded before the operation. Postoperative color Doppler ultrasound or urinary tract CTU of the transplanted kidneys were reviewed at the 1-month, 6-month, and 1 -year points after surgery, and annually thereafter, with no reobstruction or urinary leakage being observed (Figure 3). Mild vesicoureteral reflux was present in 1 patient, but no special management was required.

\section{Discussion}

Ureteral complications after renal transplantation are common and are generally difficult to solve clinic. Both microscopic intracavitary therapy and open surgery possess their respective advantages and disadvantages $(8,9)$. The former procedure is less invasive and relatively simple, 


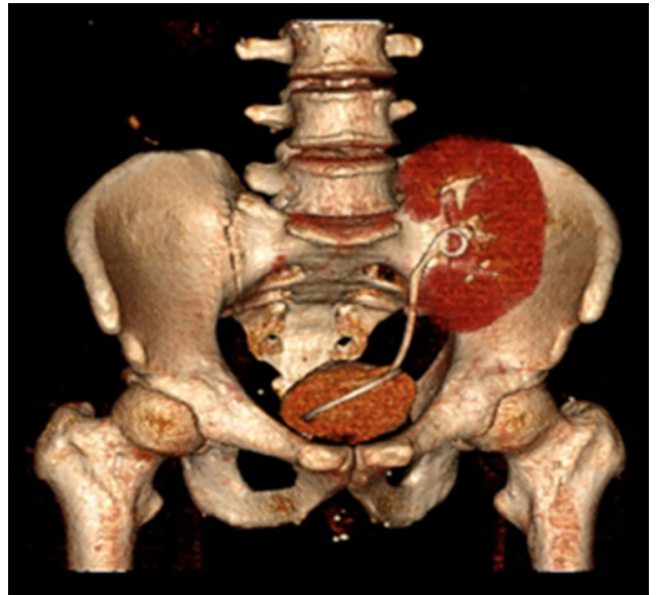

Figure 3 Computed tomography urography was performed after operation to observe the reconstruction of urinary tract.

but in most cases, requires a long-term indwelling double $\mathrm{J}$ tube, which is prone to urinary tract infection and renal graft function injury (10). The latter procedure is difficult, but after successful surgery, a long-term indwelling stent is unnecessary, and better long-term therapeutic outcomes can be achieved (11). For incomplete ureteral obstruction of the transplanted kidney, endoscopic intracavitary therapy can be conducted (5), but in most cases, the ureterocystostomy stoma's location is close to the lateral upper wall of the bladder. For patients with complete ureteral obstruction, severe urinary leakage, or even ureteral necrosis, open surgery is usually the most appropriate option. In addition to the 3 aforementioned surgical methods, other techniques, such as ureteropelvic anastomosis and renal graft lower pole anastomosis with bladder muscle flap grafting (12), can be considered, while the use of da Vinci robots for endoscopic treatment has also been reported in recent years $(13,14)$. Robotic ureteral construction has become a strong option with advantages including decreased blood loss, reducing hospital stay, and postoperative recovery compared to the open approach. While discussion of the surgical procedure not being the focus of this article, it should be noted that open surgery can be extremely difficult to perform due to anatomical alterations of the surgical area and the presence of local adhesions (15).

Adequate preoperative evaluation and preparation is essential, regardless of which surgical methods are employed. The examination and treatment process usually takes one week. All patients had a nephrostomy tube placed prior to our surgery. Pathogenic microbes were examined by culture of blood, sputum, drainage juice and urine, respectively. All patients were undergone a simultaneous urography of the upper and lower urinary tract through their nephrostomy tube and cystogram.

There are several key points relating to open surgery for the reconstruction of the urinary tract of the transplanted kidney: (I) patients with infected surgical areas should have anti-infective treatment administered until multiple negative cultures are obtained, particularly patients with urinary leakage. (II) The location of either the ureteral obstruction or urinary leakage is important, namely the length of the ureteral retention segment, as it is a critical parameter for determining the anastomosis. Generally, in both urinary obstruction and urinary leakage, the normal imaging segment of the ureter after radiography can be used as a retained ureter. A more conservative judgment should be made in the case of urinary leakage, as necrosis may occur in the development segment. (III) The degree of bladder filling is a crucial parameter in deciding the anastomosis mode. Some patients present with bladder contractures or smaller bladder volumes before renal transplantation due to long-term anuria or. for other reasons, but bladder volume can gradually return to normal following postoperative recovery of urination. However, if the urinary tract needs reconstruction prior to recovery and the bladder size is not known before surgery (for example, when performing ureterocystostomy but especially during ureterovesical muscle flap anastomosis surgery), a bladder that is too small can be the main reason for surgical failure. (IV) In patients who have undergone a transplant procedure over 2 months prior, having the transplanted kidney adhere to the surrounding tissues and the presence of fibrous repair of the entire transplantation field make freeing the ureter an arduous take, and the ureter may adhere to the arteries and veins of the transplanted kidney. Although the double J tube can be implanted preoperatively through a renal graft fistula to facilitate the intraoperative search for the ureter (16), the positioning of the ureter relative to the blood vessels remains difficult to identify, and blood vessels are prone to damage when freeing the ureter.

The key to reconstructive surgery is to fully evaluate the entire urinary tract of the transplanted kidney as well as the patient's own ipsilateral ureter before surgery. This allows for the formulation of the optimal surgical planning and for making successful preoperative predictions. The following 5 key points should be ascertained through simultaneous urography of the upper and lower urinary tract and computed tomography arteriography (CTA) and CTU 
imaging: (I) length of the transplanted kidney's developing ureteral segment, (II) bladder capacity, (III) distance between the end of the ureteral developing segment and the filled bladder, (IV) anatomical location of the ureter with surface projection markings, and $(\mathrm{V})$ positioning of the transplanted kidney's ureter relative to blood vessels.

The safety of angiography is also a major concern, particularly in the case of impaired graft function (17). Iodixanol (Visipaque $270 \mathrm{mg} / \mathrm{mL}$ ) was selected as the contrast agent, as it is isotonic and has the advantage of greatly reducing nephrotoxicity compared to hypertonic and hypotonic contrast agents (18), which reduces the occurrence of contrast-induced nephropathy. No patients in this study had a significant increase in their serum creatinine level due to the use of the aforementioned angiography method. In addition, it is of great importance that clinicians and radiologists be aware of allergy reaction caused by contrast medium. The most common symptoms of allergic reaction are rash, pruritus, headache, nausea and vomiting. Some patient even developed chest tightness, palpitations, dyspnea, decreased blood pressure.

Most experienced renal transplant surgeons perform antegrade urography of the renal graft fistula to evaluate the upper urinary tract of the transplanted kidney before performing a urinectomy (19), and in order to roughly estimate bladder volume, some surgeons may ask the patient about how much urine they usually produce. The preoperative multiangle radiography assessment this study presents provides a more visualized picture of the upper and lower urinary tract to be reconstructed as well as the positional relationship between the ureter and the vessels, which allows the surgeon to prepare their surgical plan in a more precise, safe, and reliable manner.

\section{Acknowledgments}

Funding: The work was supported by the Scientific Research Projects of Universities administered by Guangzhou (grant No. 1201630620), the Key Clinical Specialty of Guangzhou Medical University (grant No. 010004001), and Major Clinical Science and Technology Projects in Guangzhou (grant No. 2019ZD12).

\section{Footnote}

Reporting Checklist: The authors have completed the STROBE reporting checklist. Available at https://dx.doi. org/10.21037/tau-21-614
Data Sharing Statement: Available at https://dx.doi. org/10.21037/tau-21-614

Conflicts of Interest: All authors have completed the ICMJE uniform disclosure form (available at https://dx.doi. org/10.21037/tau-21-614). The authors have no conflicts of interest to declare.

Ethical Statement: The authors are accountable for all aspects of the work in ensuring that questions related to the accuracy or integrity of any part of the work are appropriately investigated and resolved. The study was conducted in accordance with the Declaration of Helsinki (as revised in 2013). The study was approved by The Institutional Review Board of the Second Affiliated Hospital of Guangzhou Medical University (number 2020hs-43) and informed consent was taken from all individual participants.

Open Access Statement: This is an Open Access article distributed in accordance with the Creative Commons Attribution-NonCommercial-NoDerivs 4.0 International License (CC BY-NC-ND 4.0), which permits the noncommercial replication and distribution of the article with the strict proviso that no changes or edits are made and the original work is properly cited (including links to both the formal publication through the relevant DOI and the license). See: https://creativecommons.org/licenses/by-nc-nd/4.0/.

\section{References}

1. Akbar SA, Jafri SZ, Amendola MA, et al. Complications of renal transplantation. Radiographics 2005;25:1335-56.

2. Woodworth TG, Furst DE. Timely renal transplantation for scleroderma end-stage kidney disease patients can improve outcomes and quality of life. Ann Transl Med 2019;7:60.

3. Alberts VP, Minnee RC, Bemelman FJ, et al. Ureteral reconstruction after renal transplantation: clinical outcome and risk factors. Urol Int 2012;88:333-7.

4. Gil-Sousa D, Oliveira-Reis D, Teves F, et al. Ureteral Stenosis After Renal Transplantation-A Single-Center 10Year Experience. Transplant Proc 2017;49:777-82.

5. Doehn C, Böse N, Meyer AJ, et al. Results of secondary ureteral implantation after kidney transplantation. Int Urol Nephrol 2011;43:669-74.

6. Asadpour A, Molaei M, Yaghoobi S. Management of ureteral complications in renal transplantation: prevention 
and treatment. Saudi J Kidney Dis Transpl 2011;22:72-4.

7. del Pizzo JJ, Jacobs SC, Bartlett ST, et al. The use of bladder for total transplant ureteral reconstruction. J Urol 1998;159:750-2; discussion 752-3.

8. Shoskes D. Renal transplantation in urology. Transl Androl Urol 2019;8:108.

9. Simsek C, Dogan SM, Piskin T, et al. Should Interventional Radiology or Open Surgery Be the First Choice for the Management of Ureteric Stenosis After Transplantation? Dual-Center Study. Transplant Proc 2017;49:517-22.

10. Kriegshauser JS, Naidu SG, Heilman RL, et al. Primary percutaneous treatment of transplant ureteral strictures using tandem stents. J Vasc Interv Radiol 2013;24:874-80.

11. Berli JU, Montgomery JR, Segev DL, et al. Surgical management of early and late ureteral complications after renal transplantation: techniques and outcomes. Clin Transplant 2015;29:26-33.

12. Gowda BD, Goldsmith P, Ahmad N. Boari flap vesicocalycostomy: a salvage drainage procedure for complete ureteric stricture and pyelocalyceal fistula. Clin Transplant 2009;23:129-31.

13. Benamran DA, Klein J, Hadaya K, et al. Post-kidney Transplant Robot-assisted Laparoscopic Ureteral (Donor-

Cite this article as: Zhang L, Liu L, Lai X, Fang J, Guo Y, Li G, Xu L, Xiong Y, Yin W, Ma J, Chen Z. Simultaneous antegrade urography of the upper urinary tract and retrograde cystography combined with computed tomography imaging in the management of ureteral complications after renal transplantation. Transl Androl Urol 2021;10(9):3620-3627. doi: 10.21037/tau-21-614 receiver) Anastomosis for Kidney Graft Reflux or Stricture Disease. Urology 2017;108:96-101.

14. Kim S, Fuller TW, Buckley JC. Robotic Surgery for the Reconstruction of Transplant Ureteral Strictures. Urology 2020;144:208-13.

15. Schult M, Küster J, Kliem V, et al. Native pyeloureterostomy after kidney transplantation: experience in 48 cases. Transpl Int 2000;13:340-3.

16. Darius T, Buemi A, Coubeau L, et al. Preperitoneal Surgical Approach to Treat Vesicoureteral Anastomotic Leakage, Distal Stenosis or Reflux After Kidney Transplantation. World J Surg 2018;42:858-65.

17. Laskey W, Aspelin P, Davidson C, et al. Nephrotoxicity of iodixanol versus iopamidol in patients with chronic kidney disease and diabetes mellitus undergoing coronary angiographic procedures. Am Heart J 2009;158:822-828.e3.

18. Kuhn MJ, Chen N, Sahani DV, et al. The PREDICT study: a randomized double-blind comparison of contrastinduced nephropathy after low- or isoosmolar contrast agent exposure. AJR Am J Roentgenol 2008;191:151-7.

19. He B, Bremner A, Han Y. Classification of ureteral stenosis and associated strategy for treatment after kidney transplant. Exp Clin Transplant 2013;11:122-7. 